\title{
Sleep-Related Eating Disorder and Nocturnal Eating Syndrome
}

\author{
Jahyeon Cho, MD¹, Ji Hyun Lee, MD¹, Seung Chul Hong, MD, PhD² \\ 'Dream Sleep Clinic, Seoul, Korea \\ 2Department of Psychiatry, St. Vincent's Hospital, College of Medicine, The Catholic University of Korea, Suwon, Korea
}

Received: December 3, 2020

Revised: December 3, 2020

Accepted: December 15, 2020

Correspondence

Seung Chul Hong, MD, PhD

Department of Psychiatry,

St. Vincent's Hospital, College of Medicine,

The Catholic University of Korea,

93 Jungbu-daero, Paldal-gu,

Suwon 16247, Korea

Tel +82-31-249-7114

Fax +82-31-248-6758

E-mail hscjohn@hotmail.com

ORCID

Jahyeon Cho

https://orcid.org/0000-0002-2230-887X

Ji Hyun Lee

https://orcid.org/0000-0001-6096-7561

Seung Chul Hong

https://orcid.org/0000-0003-0828-2906

(c) This is an Open Access article distributed under the terms of the Creative Commons Attribution Non-Commercial License (https://creativecommons.org/licenses/by-nc/4.0) which permits unrestricted non-commercial use, distribution, and reproduction in any medium, provided the original work is properly cited.
Eating and sleeping are vital functions of human life. They reciprocally influence each other in both quality and quantity. Nocturnal eating syndrome (NES) and sleep-related eating disorder (SRED) are two distinct diseases that share symptomatology of an abnormal association between the timing of eating and sleep. NES is represented by purging, a non-controllable manner of eating in the evening before sleep, followed by morning anorexia. SRED is a chronic condition showing eating episodes after sleep onset with partial to complete amnesia. Terminology was used confusedly until the third edition of the International Classification of Sleep Disorders, which defined SRED in the parasomnia section. Unlike NES, SRED usually does not respond to psychotherapy but to medication, most promisingly, to topiramate. Both are related to weight control problems, leading to obesity or excessive preoccupation with weight control issues.

Sleep Med Res 2020;11(2):65-69

Key Words Night eating syndrome, Feeding and eating disorders, Parasomnia, Sleep, Eating.

\section{INTRODUCTION}

Sleeping and eating are two of the most important activities to sustain life. They both are affected by circadian rhythms. People usually maintain the longest fasting state from the time after eating dinner, conducting activities for several hours and sleeping, to the time they wake up (7 hours to 12 hours). Chronic sleep deprivation continues to be a serious problem and the number of people who eat late at night or eat excessive amounts of food at night has increased [1].

Blood sugar levels are affected by insulin and circadian rhythms. Levels drop after eight hours of fasting during the day but are maintained at a constant level with insulin secretion during sleep [2]. Blood sugar levels are affected by sleep, so a blood sugar increase is not noticeable in a sleep-deprived state [3]. In a sleep-deprived state due to short sleep hours or sleep disorders, leptin, which controls blood sugar or appetite fails to be maintained at the proper level, resulting in abnormal increases in appetite, leading to weight gain or obesity. Sleeping and eating affect each other reciprocally under circadian and hormonal regulation.

A sleep-related eating disorder was first reported in 1955, when nocturnal eating syndrome (NES), which refers to taking more than $80 \%$ of the calories of the day at night before going to bed, was reported [4]. People with NES overeat in the evening and skip breakfast as they are rarely hungry in the morning. The circadian cycle of eating is delayed and unlike people with a sleep-related eating disorder (SRED), people with NES remember all the events in the episode. They eat mostly carbohydrate-type foods and may be overweight.

SRED involves partial confusional waking at night after falling asleep and eating. People with SRED remember none or some of the episode. As it involves behavior with increased arousal during slow-wave sleep, the third edition of the International Classification of Sleep Disorder (ICSD-3) published in 2014, classified it as sleepwalking in the non-REM (NREM)-related parasomnia section (Table 1) [5]. 
Table 1. The third edition of the International Classification of Sleep Disorders diagnostic criteria for sleep-related eating disorder

All criteria from A-D shall be satisfied.

A. Repeated non-adoptive food intake after waking up during the main sleep period

B. One or more of the following cases in relation to repeated involuntary food intake

1. When eating strange food, uneatable food or poisonous substances are consumed

2. Damage or the possibility of damage in relation to the sleep state in the process of searching for food or cooking

3. When there are negative health consequences associated with related nocturnal eating

C. There is some or complete loss of consciousness during an episode of food intake followed by impaired memory

D. There is no reasonable relationship with other sleep disorders, mental problems, medical problems, or the use of drugs or substances

\section{SLEEP-RELATED EATING DISORDER}

\section{Prevalence and Triggering Factors of SRED}

A self-reported study targeting college students reported that $4.6 \%$ of the respondents, $16.7 \%$ of the inpatient eating disorder group, and $8.7 \%$ of the outpatient eating disorder group had symptoms of SRED. Female were more likely to have SRED than male with $60-83 \%$ of the cases reported in women and the age group with the most prevalent SRED was people in their 20 s and 30s [6-9]. SRED follows a chronic course with symptoms existing for up to 15 years at the time of diagnosis [10]. Psychological stress may worsen the disease course and episodes may appear more frequently. However, it does not have a cause and effect relationship with the disease.

\section{Clinical Manifestation and Diagnosis of SRED}

Usually, SRED patients wake up and eat food and are not able to control or recognize the episodes at all. Some remember the episode if consciousness was restored while eating. However, most patients do not recall these episodes. People with partial or full memory of the event have stated that this eating behavior is difficult to control or that they have no way of controlling it. Some may seem to be completely conscious at the time of the episode but when asked about the episode the next day, they often do not remember the situation at all, just like in sleepwalking.

The foods eaten at night are mainly composed of carbohydrates such as bread and sweets. Sometimes, patients eat foods that they do not usually eat or uncooked foods (for example, frozen pizza, frozen fried rice, or chocolate). Sometimes, they carelessly use cooking utensils or the stove and do not pay attention while opening the refrigerator and removing food from it, leading to injuries and accidents. These problems can cause difficulties in controlling weight and negative health problems such as dental caries.

Mostly, people with SRED uncontrollably eat food at night, regardless of appetite. Because SRED has chronic course, the quality of sleep is chronically impaired. Sometimes, people become less self-confident because they cannot control their food intake. As they experience low self-esteem, secondary psychiatric problems such as depressive disorders or anxiety often occur. In many cases, conflicts arise as family members or spouses do not understand or deny the patient's disease and problems occur when the patients become irritable from intervention by others during the episode.

These episodes of food intake may occur once or several times a night and this night-eating pattern shows a chronic course. It is mostly found in female but occurs in male as well (it is reported that $60-83 \%$ of the patients were women). People with SRED have less appetite during the day (especially during the morning) and do not show a certain preference for what they eat while sleeping. They cannot control the food intake at night and sometimes limit food intake abnormally during the day. It is different from eating disorders such as anorexia or bulimia, which are characterized by excessive obsessive control over food.

Recently, SRED was commonly found in clinical practice in relation to the use of drugs including sleeping pills, and in this case, the patients were often unable to remember the episode and said that it was hard to control their behavior even though they are conscious at the time of the episode.

Clinical interviews with patients and family members are used to make the diagnosis. Video-assisted polysomnography (PSG) also gives information with repeated arousals and associated food or drink intake. Patients are asked to bring food during PSG testing to investigate food intake. Episodes are mainly observed in the NREM stage. If an episode of eating food is confirmed, it is possible to check not only the arousal state on the electroencephalogram at that time but also the patient's consciousness state and memory of the episode the next day. Even though when a food intake episode is not confirmed by PSG, the diagnosis cannot be excluded. Abrupt stage changes from N3 or N2 sleep to $\mathrm{W}$, as in patients with NREM parasomnia, have been observed [11]. Most of the patients have an increased arousal index during sleep and decreased sleep efficiency.

Through the PSG test, it is also possible to check whether there is an accompanying sleep disorder. The most-reported sleep problem is periodic limb movement disorder, and obstructive sleep apnea (OSA) is sometimes associated with SRED. In addition, sleepwalking is often accompanied, and even if there is no sleepwalking at the time of diagnosis, many patients have a history of sleepwalking $[8,9]$.

For this reason, SRED is regarded as a parasomnia variant, and in the ICSD-3 published in 2014, SRED is classified as one type of parasomnia [6]. 


\section{Amnesia during SRED Episodes}

In NES, there is no loss of consciousness, and eating is done while completely awake, whereas in SRED, the episode itself is often not remembered the next day. In some cases, recall, although not complete, is possible, and sometimes, patients eat food explosively even after their consciousness is fully restored or they are awake in the early stage of the episode [12]. Typically, they behave as if they are awake in other's eyes but they do not remember the episode at all the next day. However, a diagnosis of SRED cannot be excluded even though people remember the entire or part of the episode.

Whether or not remembering the episode the next day is closely related to a history of sleepwalking or a diagnosis of sleepwalking was investigated. In a study of 92 patients, $17.5 \%$ of the patients did not remember the situation, but $73 \%$ of the patients with a history of sleep-walking had at least some memory loss. People with a history of sleepwalking or those taking psychiatric drugs sometimes did not remember the entire episode $[13,14]$. The most well-documented psychiatric drug, associated with sleepwalking and SRED is zolpidem, and zolpidem-related SRED has been reported in Korea as well $[15,16]$.

\section{Treatment of SRED}

The most studied drug in relation to SRED is topiramate. The decrease in appetite from topiramate may be associated with a reduction in the number of night diet episodes along with weight loss. In a report published in $2006,68 \%$ of 30 patients treated with topiramate showed a therapeutic effect. However, most of them stopped using the drug due to side effects such as paresthesia [17]. A recent randomized controlled trial study showed that topiramate at doses from $25 \mathrm{mg}$ to $300 \mathrm{mg}$ could be used to effectively manage SRED, although many people dropped out of the study due to drug side effects [18].

In addition, the use of dopaminergic agents such as pramipexole, levodopa, and bupropion was effective in patients with restless legs syndrome (RLS) and SRED [19-21]. In SRED patients without sleepwalking, clonazepam treatment alone appeared to be ineffective. Caution should be taken as some studies reported parasomnia in patients with primary myoclonus dystonia and attention-deficit/hyperactivity disorder, and NREM parasomnia worsened after taking clonazepam and improved after suspending clonazepam [22]. Also, treatment with melatonin or agomelatine may be used in SREM as in other parasomnia disorders [23].

In contrast, patients with concomitant sleep disorders such as OSA, and secondary SERD caused by drugs, can be improved with continuous positive airway pressure treatment. If medication use like sleeping pills or antipsychotics is associated, stopping these triggering agents usually improves SRED [24].

\section{NOCTURNAL EATING SYNDROME}

NES is characterized by the excessive intake of food, especially a high carbohydrate diet, before going to bed. Psychosocial stressors are often associated and conflicts in the family may be obvious. These stressors aggravate symptoms and worsen the course of the disease. Obesity or weight gain is often found.

\section{Clinical Manifestations of NES}

According to a recent study, NES is suspected when the food consumed after dinner occupies more than $25 \%$ of the daily caloric intake [25]. It is characterized by morning anorexia since a significant amount of food is consumed during the night. As the circadian cycle is delayed, eating habits also show a delayed pattern. Even though the total amount of daily food intake is the same, those with NES consume a higher proportion of food during the evening and night.

The circadian cycle of the diet is more delayed than the circadian cycle of sleep, meaning the sleeping hours may be like those of other people while the circadian diet cycle is delayed [26].

A study comparing behavioral and neuroendocrine circadian rhythms (glucose, insulin, ghrelin, leptin, melatonin, cortisol, thyroid-stimulating hormone, and prolactin) in normal people and NES patients showed significant changes in timing and the amplitude of meal timing, circadian rhythms for total caloric, fat, and carbohydrate intake, and physiological circadian markers involved in appetite and neuroendocrine regulation [27].

The kinds of food are not much different from the food eaten during the day, but carbohydrates account for a high proportion of the diet. Carbohydrate-rich food accounts for a higher proportion of the food consumed in the evening compared to food consumed during the day. There is also a report that overweight or obesity tends to occur, and the pattern of evening hyperphagia is worsened due to obesity. Most NES patients have no appetite during the morning, skip breakfast, seldom eat, or eat the first meal of the day very late.

No firm diagnostic criteria have been established. The limitation of previous studies is that various diagnostic criteria are used. The recent consensus includes a daily eating pattern of significantly increased intake in the evening and/or nighttime, as manifested by one or both of the following: at least $25 \%$ of the food intake is consumed after the evening meal or at least two episodes of nocturnal eating per week. An important recent addition to the core criteria included the presence of significant distress and/ or impairment in functioning [28].

\section{Treatment of NES}

NES is thought to be an abnormality in the regulation of carbohydrate intake during the night and is associated with abnormal serotonin regulation. Studies have mainly focused on the effect of selective serotonin reuptake inhibitors (SSRIs), and ser- 
traline at doses between 50-200 mg have been well-studied. A weight-loss effect from sertraline was demonstrated in overweight or obese patients [29-31].

In NES patients, the use of sleeping pills such as zolpidem was not effective. Moreover, zolpidem should be used with caution because it can be associated with SRED in patients with or without NES. Cognitive-behavioral therapy using cognitive therapy components such as identifying, evaluating, and restructuring maladaptive thoughts, with a behavioral weight loss component (monitoring food consumption, regulating meals and snacks, and restricting caloric intake), shifting food intake to earlier in the day, and interrupting the relationship between nighttime eating and sleep onset has been used [32]. Since circadian phase delay is associated with NES, efforts to advance circadian rhythm such as bright light therapy have been tried [33].

\section{SLEEP-RELATED EATING DISORDER AND NOCTURNAL EATING SYNDROME}

Both diseases have unique eating intake related to sleep (Table 2). NES usually involves excessive food intake mainly after dinner until going to sleep (evening hyperphagia). The neuroendocrine system including physiological melatonin levels and appetite-controlling hormones as well as mealtime and food intake patterns including total calories, carbohydrates, and fat consumption patterns shows circadian delays. Patients with NES may complain of poor sleep quality, which can be a secondary effect of eating too late and sleep initiation difficulty associated with the delay in physiological sleep time. The patients sometimes have cognitive distortions, manifesting as the inability to sleep if they feel hungry. However, they do not show habitual eating patterns while sleeping like people with SRED. Treatment using cognitive reconstruction and behavioral modification has been tried. SSRIs such as sertraline showed improvement in the psychiatric profile as well as successful body weight control.

The main symptomatology of patients with SRED is eating after sleep onset with partial to no recall. They have impaired quality of sleep. However, it is directly associated with the disruption of the normal sleep cycle pattern. Abrupt sleep stage changes are also seen in parasomnia. Medications like hypnotics and antipsychotics are known to be associated with SRED. Treatment of underlying sleep problems such as RLS and OSA can improve the symptoms of SRED.

Problems with weight gain and obesity are also seen in patients with NES and SRED. Medical problems such as hyperlipidemia, hypercholesterolemia, and obesity are also seen.

\section{Conclusion}

SRED and NES are diseases that can cause secondary psychiatric disorders. They take a chronic course, leading to weight gain or obesity. NES is a problem in the circadian cycle of the diet compared to the circadian cycle of sleep and can be improved by the administration of sertraline. SRED is a problem of eating while sleeping and can be treated with topiramate if well-tolerated. In SRED patients, it is necessary to conduct PSG studies to identify other coexisting sleep disorders and if other sleep disorders such as RLS, periodic limb disorders, or sleep apnea syndrome are diagnosed, the treatment of such disorders may improve SRED symptoms. Further discussion is necessary since there are no firmly established NES diagnostic criteria. SRED associated or not-associated with medication shows a chronic pattern that is difficult to manage. Those with hypnotic-drug associated SRED can be best managed with cognitive behavioral therapy for insomnia (CBT-I). However, patients with both in-

Table 2. SRED and NES

\begin{tabular}{|c|c|c|}
\hline & NES & SRED \\
\hline Time & In the evening before bedtime & After sleep onset \\
\hline Memory of the episode & Complete recall of the episode & From complete recall to partial recall to no remembrance \\
\hline Type of food & High carbohydrates such as bread, pizza, and cake, etc. & $\begin{array}{l}\text { From high carbohydrates to uncooked food which is not } \\
\text { usually eaten }\end{array}$ \\
\hline $\begin{array}{l}\text { Comorbid medical or } \\
\text { psychiatric condition }\end{array}$ & $\begin{array}{l}\text { Depression, eating disorder, obesity, } \\
\text { hypercholesterolemia }\end{array}$ & Depression, obesity, hypercholesterolemia \\
\hline $\begin{array}{l}\text { Concomitant circadian } \\
\text { or sleep disorder }\end{array}$ & $\begin{array}{l}\text { Delayed circadian phase syndrome is sometimes } \\
\text { observed }\end{array}$ & Sleepwalking, restless legs syndrome \\
\hline Associated drugs & Not known & $\begin{array}{l}\text { Hypnotics (zolpidem, triazolam), antipsychotics (usually } \\
\text { atypical antipsychotics like olanzapine, quetiapine, } \\
\text { risperidone, and aripiprazole) }\end{array}$ \\
\hline Management & Sertraline, cognitive behavioral therapy & $\begin{array}{l}\text { Topiramte, benzodiazepine (clonazepam), dopaminergic } \\
\text { agents like pramipexole, bupropion-levodopa-trazodone, } \\
\text { melatonin, and agomelatine }\end{array}$ \\
\hline
\end{tabular}

SRED: sleep-related eating disorder, NES: nocturnal eating syndrome. 
somnia and hypnotic-drug associated with SRED are less compliant with CBT-I than those without SRED even though there is little documentation regarding the effectiveness of CBT-I in hypnotic drug-associated SRED. SRED usually shows a chronic pattern, causing intrapsychic stress and intra-familial conflict. More clinical attention is needed in managing these conditions, and uncontrollable eating associated with a particular time of the day (evening and during sleep) needs further clinical attention.

\section{Acknowledgments}

We thank Professor Christian Guilleminault at Stanford University, who guided countless sleep specialists worldwide.

\section{Conflicts of Interest}

The authors have no financial conflicts of interest.

\section{Authors' Contribution}

Conceptualization: Cho J. Investigation: Lee JH. Resources: Lee JH. Supervision: Hong SC. Validation: Hong SC. Writing_original draft: Cho J, Lee JH. Writing—review \& editing: Cho J, Lee JH.

\section{REFERENCES}

1. Chung S, Cho SW, Jo MW, Youn S, Lee J, Sim CS. The prevalence and incidence of insomnia in Korea during 2005 to 2013. Psychiatry Investig 2020;17:533-40.

2. Knutson KL. Impact of sleep and sleep loss on glucose homeostasis and appetite regulation. Sleep Med Clin 2007;2:187-97.

3. Trenell MI, Marshall NS, Rogers NL. Sleep and metabolic control: waking to a problem? Clin Exp Pharmacol Physiol 2007;34:1-9.

4. Stunkard AJ, Grace WJ, Wolff HG. The night-eating syndrome; a pattern of food intake among certain obese patients. Am J Med 1955;19: 78-86.

5. American Academy of Sleep Medicine. International classification of sleep disorders. 3rd ed. Darien: American Academy of Sleep Medicine 2014;240-5.

6. Winkelman JW, Herzog DB, Fava M. The prevalence of sleep-related eating disorder in psychiatric and non-psychiatric populations. Psychol Med 1999;29:1461-6.

7. Schenck CH, Mahowald MW. Review of nocturnal sleep-related eating disorders. Int J Eat Disord 1994;15:343-56.

8. Winkelman JW. Clinical and polysomnographic features of sleep-related eating disorder. J Clin Psychiatry 1998;59:14-9.

9. Vetrugno R, Manconi M, Ferini-Strambi L, Provini F, Plazzi G, Montagna P. Nocturnal eating: sleep-related eating disorder or night eating syndrome? A videopolysomnographic study. Sleep 2006;29:949-54.

10. Howell MJ, Schenck CH, Crow SJ. A review of nighttime eating disorders. Sleep Med Rev 2009;13:23-34.

11. Cho J, Lee JH. Considering eating behaviors at night: sleep related eating disorder. Bulletin of Center for Clinical Psychology Kinki University 2011;4:11-8.

12. Winkelman JW. Sleep-related eating disorder and night eating syndrome: sleep disorders, eating disorders, or both? Sleep 2006;29:876-7.

13. de Zwaan M, Roerig DB, Crosby RD, Karaz S, Mitchell JE. Nighttime eating: a descriptive study. Int J Eat Disord 2006;39:224-32.
14. Whyte J, Kavey NB. Somnambulistic eating: a report of three cases. Int J Eat Disord 1990;9:577-81.

15. Hwang J, Lee GH, Lee SA. A case of zolpidem-induced sleep-related eating disorder. J Korean Sleep Res Soc 2012;9:15-8.

16. Cho JW, Jung KY. A case with sleep related eating disorder. J Korean Sleep Res Soc 2008;5:100-2.

17. Winkelman JW. Efficacy and tolerability of open-label topiramate in the treatment of sleep-related eating disorder: a retrospective case series. J Clin Psychiatry 2006;67:1729-34.

18. Winkelman JW, Wipper B, Purks J, Mei L, Schoerning L. Topiramate reduces nocturnal eating in sleep-related eating disorder. Sleep 2020; 43:zsaa060.

19. Provini F, Albani F, Vetrugno R, Vignatelli L, Lombardi C, Plazzi G, et al. A pilot double-blind placebo-controlled trial of low-dose pramipexole in sleep-related eating disorder. Eur J Neurol 2005;12:432-6.

20. Kobayashi N, Yoshimura R, Takano M. Successful treatment with clonazepam and pramipexole of a patient with sleep-related eating disorder associated with restless legs syndrome: a case report. Case Rep Med 2012;2012:893681.

21. Schenck CH, Mahowald MW. Combined bupropion-levodopa-trazodone therapy of sleep-related eating and sleep disruption in two adults with chemical dependency. Sleep 2000;23:587-8.

22. Ghosh D, Petrecca AM, Khuhro AL. Sleep-related eating disorder (SRED): paradoxical effect of clonazepam. J Clin Sleep Med 2018;14: 1261-3.

23. Zapp AA, Fischer EC, Deuschle M. The effect of agomelatine and melatonin on sleep-related eating: a case report. J Med Case Reports 2017; 11:275.

24. Chiaro G, Caletti MT, Provini F. Treatment of sleep-related eating disorder. Curr Treat Options Neurol 2015;17:361.

25. Allison KC, Ahima RS, O'Reardon JP, Dinges DF, Sharma V, Cummings DE, et al. Neuroendocrine profiles associated with energy intake, sleep, and stress in the night eating syndrome. J Clin Endocrinol Metab 2005;90:6214-7.

26. O’Reardon JP, Ringel BL, Dinges DF, Allison KC, Rogers NL, Martino NS, et al. Circadian eating and sleeping patterns in the night eating syndrome. Obes Res 2004;12:1789-96.

27. Goel N, Stunkard AJ, Rogers NL, Van Dongen HP, Allison KC, O’Reardon JP, et al. Circadian rhythm profiles in women with night eating syndrome. J Biol Rhythms 2009;24:85-94.

28. Cleator J, Abbott J, Judd P, Sutton C, Wilding JP. Night eating syndrome: implications for severe obesity. Nutr Diabetes 2012;2:e44.

29. Stunkard AJ, Allison KC, Lundgren JD, Martino NS, Heo M, Etemad $\mathrm{B}$, et al. A paradigm for facilitating pharmacotherapy at a distance: sertraline treatment of the night eating syndrome. J Clin Psychiatry 2006; 67:1568-72.

30. O'Reardon JP, Allison KC, Martino NS, Lundgren JD, Heo M, Stunkard AJ. A randomized, placebo-controlled trial of sertraline in the treatment of night eating syndrome. Am J Psychiatry 2006;163:893-8.

31. O'Reardon JP, Stunkard AJ, Allison KC. Clinical trial of sertraline in the treatment of night eating syndrome. Int J Eat Disord 2004;35:16-26.

32. Allison KC, Lundgren JD, Moore RH, O'Reardon JP, Stunkard AJ. Cognitive behavior therapy for night eating syndrome: a pilot study. Am J Psychother 2010;64:91-106.

33. McCune AM, Lundgren JD. Bright light therapy for the treatment of night eating syndrome: a pilot study. Psychiatry Res 2015;229:577-9. 